\title{
First-Principles Study of Graphene-6H SiC Surface Interactions
}

\author{
Ahmet Cicek and Bulent Ulug
}

\begin{abstract}
Interactions of graphene with $6 \mathrm{H}-\mathrm{SiC}\{0001\}$ surfaces are numerically investigated from first principles. In order to describe the bulk structure and its 6 bilayer thick surfaces correctly, bare and dipole-corrected atomic relaxations are considered. The obtained lattice parameters and bulk modulus are in good agreement with experimental values. The calculated indirect band gap width of $2.10 \mathrm{eV}$ is smaller than the experimental value due to the nature of the computational method. Geometrical optimization of the surfaces, where dipole correction is applied, reveals that the first two bilayers displace significantly, where the relaxations of the very top bilayer is more pronounced. Band structures of the $\{0001\}$ surfaces possess two flat bands around the Fermi level due to unsaturated bonds on opposite faces. When one layer of $\mathrm{C}$ atoms are introduced on the Si-terminated surface, it behaves as a tightly-bound buffer layer. This is also the case for the $\mathrm{C}$-terminated surface when van der Waals interactions are taken into account. In contrast, disregarding these interactions yields free-standing graphene like behavior for the first $\mathrm{C}$ overlayer. On both surfaces, the second $\mathrm{C}$ overlayer is free-standing where the corresponding band structures incorporate Dirac-cone like features.
\end{abstract}

Index Terms-Graphene, $\mathbf{6 H}$ Silicon Carbide, Density Functional Theory, Surface analysis, Band structure

\section{INTRODUCTION}

G RAPHENE, monolayer of graphite, was first obtained in 2004 by mechanical exfoliation from graphite [1]. Since then, it has been extensively studied both theoretically and experimentally. Theoretically it has been drawing attention due to phenomena such as observation of quantum Hall effect at room temperature [2] and the Klein paradox, a phenomenon important in the context of relativity [3]. Due to its superior mechanical, electronic and optical properties, it has been finding applications in device physics $[4,5]$.

Among the most common methods for obtaining few-layer graphene are mechanical exfoliation from graphite [1], arc discharge [6] and intercalation of graphite by active elements

AHMET CICEK, is with Department of Nanoscience and Nanotechnology, Burdur Mehmet Akif Ersoy University, Burdur, Turkey, (e-mail: ahmetcicek@mehmetakif.edu.tr).

\section{iD https://orcid.org/0000-0002-7686-0045}

BULENT ULUG, is with Department of Physics, Akdeniz University, Antalya, Turkey, (e-mail: bulug@akdeniz.edu.tr).

iD https://orcid.org/0000-0003-1744-6861

Manuscript received April 1, 2021; accepted April 27, 2021.

DOI: $10.17694 /$ bajece. 908183 like $\mathrm{Li}$ and $\mathrm{K}$ [7]. However, epitaxial growth on substrates such as silicon carbide ( $\mathrm{SiC}$ ) with $8 \%$ lattice mismatch [8] and boron nitride $(\mathrm{BN})$ with $2 \%$ lattice mismatch [9] are preferred for large-scale crystal quality growth of graphene. In addition, epitaxial graphene growth can also be achieved on metallic substrates such as nickel $(\mathrm{Ni})$, cobalt $(\mathrm{Co})$, copper $(\mathrm{Cu})$, rubidium $(\mathrm{Ru})$ and platinum $(\mathrm{Pt})$ [10-14]. Although highquality monolayer (ML) graphene can be grown on metallic substrates, they are not suitable for microelectronic applications due to charge transfer to graphene. In contrast, $\mathrm{SiC}$ with approximately $3 \mathrm{eV}$ band gap is more suitable for epitaxial graphene growth. Especially $\{0001\}$ surfaces of hexagonal $4 \mathrm{H}-\mathrm{SiC}[15-17]$ or $6 \mathrm{H}-\mathrm{SiC}[18,19]$ are preferred for high-quality graphene growth.

Graphene exhibits different physical properties on opposite surfaces of hexagonal $\mathrm{SiC}$ terminated by $\mathrm{Si}$ or $\mathrm{C}$ atoms [20, 21]. Thus, understanding of graphene-substrate interactions is vital for design of graphene based nanodevices and investigation of their physical properties. For instance, the first graphene layer grown on Si-terminated face of $\mathrm{SiC}$ is tightly bound to surface, which has a complex reconstruction, while free-standing graphene layers are obtained starting from the second layer [20]. In contrast, the first layer grown on the Cterminated face of $\mathrm{SiC}$ is free standing, where there exist rotational defects between graphene layers [22].

Considering the complex surface reconstruction of hexagonal $\mathrm{SiC}$, first numerical studies employing densityfunctional theory (DFT) calculations on graphene-SiC interactions involved relatively small surface reconstructions $[8,23,24]$. Although these studies suggested that the very first layer on both Si-terminated and C-terminated surfaces were tightly-bound buffer layers, more recent studies indicate that the buffer layer does not appear on the C-terminated face [25, 26]. Thus, it is important to understand the correct behavior of graphene on opposite faces of hexagonal $\mathrm{SiC}$.

In this work, graphene-substrate interactions for the commonly employed $6 \mathrm{H}-\mathrm{SiC}\{0001\}$ surfaces are numerically investigated from first principles via DFT calculations. Structural and electronic properties of graphene on opposite surfaces are compared to understand the formation mechanism and physical properties of graphene on these surfaces.

\section{MATERIAL AND METHODS}

Crystal structure of the $6 \mathrm{H}$ silicon carbide substrate in the $\mathrm{S}_{3}$ configuration [27] is seen in Fig. 1(a). Here, each carbon (silicon) atom is tetravalently bonded to four silicon (carbon) atoms, where the bond length is $1.89 \AA$ and the $\mathrm{C}$-Si-C bond angle is 109.57 degrees. It is designated $6 \mathrm{H}$ since its unit cell 
in the hexagonal crystal class comprises 6 bilayers (BLs) in the ABCACB... stacking order [28]. The Si-C bonds follow a zig-zag pattern over the (11̄20) plane shown in Fig. 1(b). Positions of the atoms on this plane are denoted as $z_{i}$ $(i=1 \ldots 12)$ (Fig. 1(c)), whereas the vertical distances in a BL and between two BLs are denoted as $d_{i}$ and $h_{i}$, respectively. Thus, $6\left(d_{i}+h_{i}\right)=c$, lattice constant in the [0001] ( $z$ ) direction. The first Brillouin zone for $6 \mathrm{H} \mathrm{SiC}$ and the high-symmetry points over the $\{0001\}$ surfaces are given in Fig. 1(d).
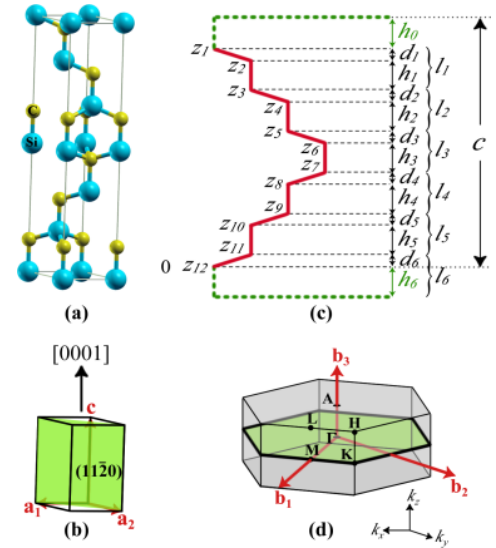

Fig.1. The crystal structure of $6 \mathrm{H} \mathrm{SiC} \mathrm{(a),} \mathrm{definition} \mathrm{of} \mathrm{(0001)} \mathrm{direction} \mathrm{and}$ $(11 \overline{2} 0)$ plane (b), definition of atomic positions over the $(11 \overline{2} 0)$ plane (c) and the first Brillouin zone, as well as high-symmetry points for $\{0001\}$ surfaces.

Graphene is grown on $6 \mathrm{H} \mathrm{SiC}\{0001\}$ surfaces. Here, the (0001) surface is terminated by a sub-layer composed of only $\mathrm{Si}$ atoms, whereas the $(000 \overline{1})$ surface is terminated by a $\mathrm{C}$ sublayer. Growth on $6 \mathrm{H} \mathrm{SiC} \mathrm{takes} \mathrm{place} \mathrm{through} \mathrm{a} \mathrm{thermal}$ process where $\mathrm{Si}$ atoms sublime and the remaining $\mathrm{C}$ atoms on the very top of the surface form surface reconstructions [29]. The most common surface reconstruction for epitaxial graphene growth is denoted as $6 \sqrt{3} \times 6 \sqrt{3} R 30^{\circ}[30]$. However, in this study a more computationally afordable surface reconstruction of $\sqrt{3} \times \sqrt{3} R 30^{\circ}$ is considered $[8,23]$.

The top view of the (0001) surface of $6 \mathrm{H} \mathrm{SiC}$ is seen in Fig. 2(a), where the enumeration of top $\mathrm{BL}$ atoms is also shown. Also in Fig. 2(a) is the graphene ML considered on $6 \mathrm{H} \mathrm{SiC}$ surface and the enumeration of $\mathrm{C}$ atoms in this layer.

Geometry of a graphene ML on $\sqrt{3} \times \sqrt{3} R 30^{\circ}$ reconstruction of $6 \mathrm{H} \mathrm{SiC}$ is on the right of Fig. 2(a). The side view of the geometry under study for the case of (0001) surface is given in Fig. 2(b). Here, a BL graphene is demonstrated, where $d_{G A}$ and $d_{G G}$ are graphene-substrate and graphene-graphene distances, respectively. In the case of graphene $\mathrm{BL}$, the $\mathrm{ABAB} \ldots$ stacking order is considered.

The numerical problems in Fig. 1 and Fig. 2 are three dimensional (3D) in nature. However, the study of solid surfaces requires termination of an otherwise infinite system along a given direction. While the unit cell in Fig. 1(a) defined with Bloch periodic boundary condition is sufficient for the study of bulk crystals, study of surfaces requires supercell structures, where the finite-size crystal is surrounded by a vacuum layer of thickness $d_{v a c}\left(h_{0}\right.$ and $h_{6}$ in Fig. 1(c)).

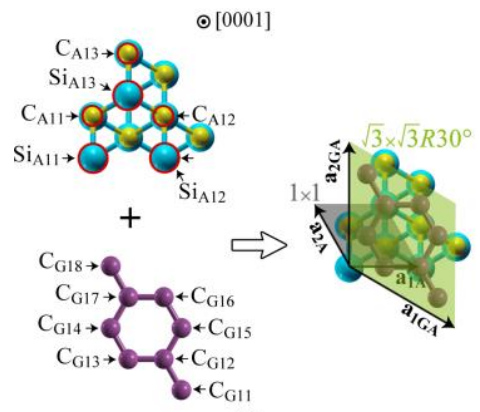

(a)

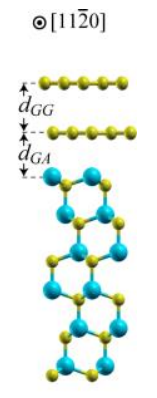

(b)
Fig.2. The $\sqrt{3} \times \sqrt{3} R 30^{\circ}$ model to investigate graphene- $6 \mathrm{H} \mathrm{SiC}$ interactions (a) and side view of the substrate-graphene BL model along the $[11 \overline{2} 0]$ direction (b).

In this study, an asymmetric supercell, as seen in Fig. 3, due to electrical polarity of the $6 \mathrm{H} \mathrm{SiC}\{0001\}$ surfaces is considered. Since the $\mathrm{Si}$ and $\mathrm{C}$ atoms on opposite faces have unequal electron affinities, a net surface charge density develops [31]. For large number of cells, this polarization can be ignored. However, for small supercells as in Fig. 3, ignoring it may result in significant error.

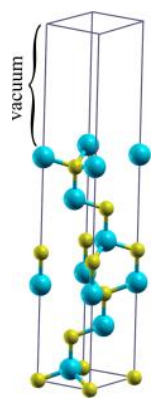

Fig.3. The numerical model employed for the investigation of $6 \mathrm{BL}$-thick $6 \mathrm{H}$ SiC $\{0001\}$ surfaces.

Graphene-6H SiC interactions are numerically investigated from first principles (ab-initio) via DFT calculations implemented in Quantum Espresso software [32]. This software employs a plane-wave basis set for the calculation of density functionals from Kohn-Sham equations. The local density approximation (LDA) and generalized gradient approximation (GGA) for the exchange-correlation functionals are utilized. Perdew-Zunger (1981) parametrization [33] in case of LDA and Perdew-Wang 91 (PW91) parametrization [34] for GGA are employed.

Brillouin zone integrals are discretized through MonkhorstPack (MP) [35] k-point meshes. In case of bulk $6 \mathrm{H} \mathrm{SiC}$ and its $\{0001\}$ surfaces, a $12 \times 12 \times 2$ MP grid is used. In free-standing graphene calculations, 6x6x6 MP grids are employed. In addition, the cut-off energy in all calculations is $50 \mathrm{Ry}$.

The calculations are conducted in four stages. First, the equilibrium geometry for bulk $6 \mathrm{H} \mathrm{SiC}$ in Fig. 1(a) is obtained through unconstrained geometric relaxation. Here, the convergence criterion is reduction of Helmann-Feynman forces below $2.0 \times 10^{-4} \mathrm{Ry} / \mathrm{Bohr}\left(5.1 \times 10^{-3} \mathrm{eV} / \AA ̊\right)$. In addition, the band structure of bulk $6 \mathrm{H} \mathrm{SiC}$ is obtained. In the second 
stage, $6 \mathrm{H} \mathrm{SiC}\{0001\}$ surfaces are geometrically optimized. Here, the 2D model in Fig. 1(c) is considered. In each case, 3 of 6 BLs are relaxed, while the rest are fixed. The vacuum layer thickness in these calculations is adopted as $d_{v a c}=20 \AA$.

\section{RESULTS AND DISCUSSION}

Numerically-obtained lattice constants $(a$ and $c$ ) from the Birch-Murnaghan equation of state fitting [36] are given in Table 1. They are in good agreement with both experimental [37-39] and previous numerical [28, 40-42] data. However, LDA approach resulted in a slightly closer value to the experimental value of $a=3.081 \AA$ [37]. In addition, the bulk modulus $\left(B_{0}\right)$ values are also close to the experimentallyobtained values [38, 39], while GGA approach yields a closer value. Besides, the derivative of bulk modulus $\left(B_{0}{ }^{\prime}\right)$ is considerably larger than the experimental value [39].

TABLE 1

COMPARISON OF THE LATTICE AND ELASTIC PARAMETERS OBTAINED IN THIS WORK WITH THE VALUES IN THE LITERATURE.

\begin{tabular}{lcclr}
\hline \hline \multirow{2}{*}{ Parameter } & LDA & GGA & Experimental & Numerical \\
\hline$a(\AA)$ & 3.094 & 3.050 & $3.081^{\mathrm{a}}$ & $3.077^{\mathrm{d}}(\mathrm{LDA})$ \\
& & & & $3.033^{\mathrm{e}}(\mathrm{GGA})$ \\
& & & & $3.099^{\mathrm{f}}(\mathrm{GGA})$ \\
& & & & $3.095^{\mathrm{g}}(\mathrm{GGA})$ \\
$c / a$ & 4.909 & 4.908 & $4.909^{\mathrm{a}}$ & $4.910^{\mathrm{d}}(\mathrm{LDA})$ \\
& & & & $4.906^{\mathrm{e}}(\mathrm{GDA})$ \\
& & & & $4.908^{\mathrm{f}}(\mathrm{LDA})$ \\
& & & & $4.907^{\mathrm{g}}(\mathrm{GGA})$ \\
$B_{0}(\mathrm{GPa})$ & 210.94 & 228.94 & $230.2( \pm 4.0)^{\mathrm{b}}$ & \\
& & & $260( \pm 9)^{\mathrm{c}}$ & \\
$B_{0}{ }^{{ }^{\mathrm{C}}}$ & 3.92 & 4.57 & $2.9( \pm 0.3)^{\mathrm{c}}$ & \\
\hline \hline
\end{tabular}

${ }^{\mathrm{a}}$ Ref. [37], ${ }^{\mathrm{b}}$ Ref. [38], ${ }^{\mathrm{c}}$ Ref. [39], ${ }^{\mathrm{d}}$ Ref. [28], ${ }^{\mathrm{e}}$ Ref. [40], ${ }^{\text {Ref. [41], }}$ gRef. [42]

Results of geometrical relaxations of the bulk $6 \mathrm{H} \mathrm{SiC}$ indicates that the relaxations exhibit repeating behavior with 3 BL periods. Therefore, $d_{i+3}=d_{i}$ and $h_{i+3}=h_{i}(i=1,2,3)$. The values of $d_{i}$ and $h_{i}$ in Table 2 are in good agreement with the results of Käckell et al [40]. Overall, the atoms experience relaxations around $1 \mathrm{pm}$.

TABLE 2

CALCULATED DISTANCES WITHIN AND BETWEEN BILAYERS FOR BULK 6H SILICON CARBIDE

\begin{tabular}{lccccc}
\hline \hline & \multicolumn{2}{c}{ Fixed atoms } & \multicolumn{2}{c}{ Relaxed } & Literature \\
& LDA & GGA & LDA & GGA & (Ref. [40], LDA) \\
\cline { 2 - 6 }$d_{1}(\AA)$ & $\mathbf{0 . 6 2}$ & 0.63 & $\mathbf{0 . 6 3}$ & 0.64 & 0.63 \\
$h_{1}(\AA)$ & $\mathbf{1 . 8 7}$ & 1.90 & $\mathbf{1 . 8 7}$ & 1.90 & 1.86 \\
$d_{2}(\AA)$ & $\mathbf{0 . 6 2}$ & 0.63 & $\mathbf{0 . 6 2}$ & 0.63 & 0.62 \\
$h_{2}(\AA)$ & $\mathbf{1 . 8 7}$ & 1.90 & $\mathbf{1 . 8 7}$ & 1.90 & 1.86 \\
$d_{3}(\AA)$ & $\mathbf{0 . 6 2}$ & 0.63 & $\mathbf{0 . 6 2}$ & 0.63 & 0.62 \\
$h_{3}(\AA)$ & $\mathbf{1 . 8 7}$ & 1.90 & $\mathbf{1 . 8 8}$ & 1.90 & 1.87 \\
\hline \hline
\end{tabular}

The band structure of geometrically optimized $6 \mathrm{H} \mathrm{SiC}$ is given in Fig. 4. Here, an indirect band gap of $E_{G}=2.10 \mathrm{eV}$ between the $\Gamma$ and $\mathrm{L}$ points is obtained through LDA calculations, which is considerably smaller than the experimental value of $3.08 \mathrm{eV}$ [43]. This is a well-known underestimation in LDA band structure calculations [44]. A similar result is obtained for GGA calculations. To calculate the band gap correctly, hybrid functionals are utilized, which are not considered in this work.

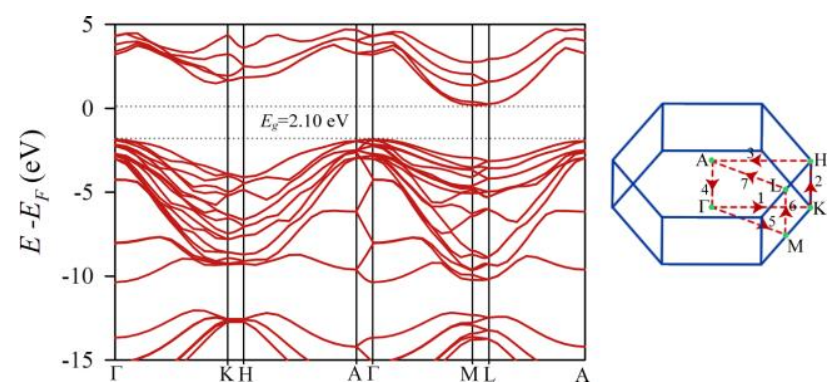

Fig.4. The band structure of $6 \mathrm{H} \mathrm{SiC}$ through LDA calculations. The k-path is given in the first Brillouin zone on the right.

Since investigation of graphene-substrate interactions involves surfaces, the projection of the bulk band structure in Fig. 4 over the $1 \times 1\{0001\}$ surface is also investigated. Reduction of dimension is achieved by varying the $z$ component of the wave vector $\left(k_{z}\right)$ between 0 and $\pi / c$ and calculating the corresponding band structure for each $k_{z}$ over the ГМКГ path seen in Fig. 4. The final projected band structure given in Fig. 5 is obtained through superposition of all individual band structures. The $6 \mathrm{H}-\mathrm{SiC}\{0001\}$ surface bands are overlaid on this projected band structure to understand the effects of surface termination. In addition to the $2.10 \mathrm{eV}$ band gap, pocket band gaps localized in the valence and conduction bands [23], as well as a stop band around $E-E_{F}=-10 \mathrm{eV}$ are observed in Fig. 5.

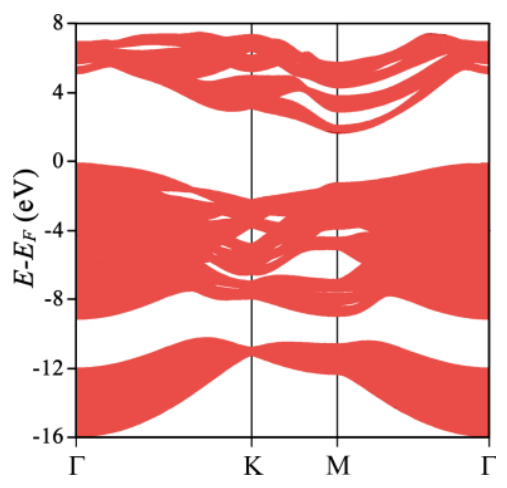

Fig.5. Projection of bulk $6 \mathrm{H} \mathrm{SiC}$ electronic bands over the $1 \times 1\{0001\}$ surface.

By employing the supercell in Fig. 3 and dipole correction, geometrical optimization of $6 \mathrm{H} \mathrm{SiC} \mathrm{(0001)} \mathrm{(Si} \mathrm{terminated)} \mathrm{and}$ $(000 \overline{1})(C$ terminated) surfaces are carried out. In both cases, only the first $3 / 6$ BLs are relaxed and the variations of the $d_{i}$ and $h_{i}$ values with respect to the corresponding values in bulk $6 \mathrm{H} \mathrm{SiC}$ are calculated. The results are presented in Table 3. It is clearly seen that the relaxation is largest in the top BL for both cases. Percent variations of $d_{i}$ and $h_{i}$ for the second BLs are considerably smaller, Table 3 . Moreover, relaxations in the 
third BLs are negligible. Besides, for relaxation from the $\mathrm{Si}$ terminated surface, LDA calculations yield values almost twice of the values obtained through GGA calculations. Closer inspection of Table 3 reveals that relaxation of the first $\mathrm{C}$ sublayer on the $(000 \overline{1})$ surface is relatively higher. This is related to the charge transfer towards the $(000 \overline{1})$ side, whose polarity is higher.

TABLE 3

PERCENT VARIATONS OF THE DISTANCES WITHIN AND BETWEEN BILAYERS FOR 6 H SILICON CARBIDE $\{0001\}$ SURFACES WITH RESPECT TO THE VALUES FOR THE BULK CRYSTAL

\begin{tabular}{|c|c|c|c|}
\hline & $\begin{array}{l}\text { Distance } \\
\text { Variation }\end{array}$ & LDA & GGA \\
\hline \multirow{4}{*}{ 汤 } & $\Delta d_{1}(\%)$ & -6.8 & -3.2 \\
\hline & $\Delta h_{1}(\%)$ & 1.0 & 0.6 \\
\hline & $\Delta d_{2}(\%)$ & -1.6 & -0.5 \\
\hline & $\Delta h_{2}(\%)$ & 0.5 & 0.3 \\
\hline \multirow{4}{*}{ ڤె } & $\Delta d_{6}(\%)$ & -37.4 & -33.3 \\
\hline & $\Delta h_{6}(\%)$ & 4.3 & 3.6 \\
\hline & $\Delta d_{5}(\%)$ & -5.7 & -3.5 \\
\hline & $\Delta h_{5}(\%)$ & 1.0 & 0.8 \\
\hline
\end{tabular}

Band structures of the $6 \mathrm{H} \mathrm{SiC}\{0001\}$ surfaces overlaid on the projected bulk bands are given in Fig. 6. The presented band structures are obtained through LDA calculations by considering the relaxed surfaces. The Fermi level $\left(E_{F}\right)$ is above the valence band maximum of the bulk $(0 \mathrm{eV})$ by 0.5 $\mathrm{eV}$ and $0.3 \mathrm{eV}$ for the $\mathrm{Si}$ - and C-terminated surfaces, respectively. In both cases, two surface bands localized around $E_{F}$ are observed, Figure 6 . These bands originate from the dangling $\mathrm{p}$ states of the $\mathrm{Si}_{1}$ and $\mathrm{C}_{6}$ atoms on the opposite faces. The band due to $\mathrm{C}_{6}$ is flat since this side is more planar [45]. In addition, a single band in the stop band around $-10 \mathrm{eV}$ is also seen for both surfaces, Fig. 6.

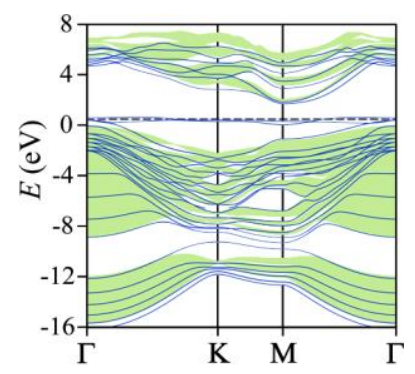

(a)

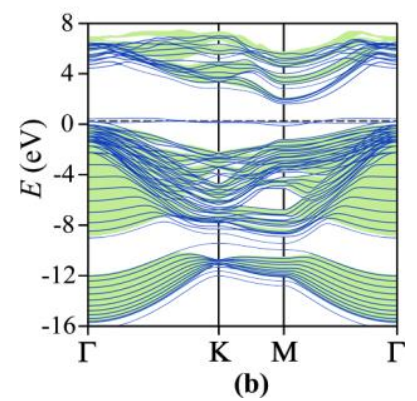

Fig.6. Band structures of the $6 \mathrm{H} \mathrm{SiC} \mathrm{(0001)} \mathrm{(a)} \mathrm{and} \mathrm{(000} \overline{1})$ surfaces overlaid on the projected bands of the bulk. The horizontal dashed lines denote the Fermi levels $\left(E_{F}\right)$.
The band structures of ML and BL graphene are given in Fig. 7. Since results of LDA and GGA calculations agree well, only the results of LDA calculations will be given in the following band structures. Fig. 7(a) shows that the $E_{F}$ and Dirac point $\left(E_{D}\right)$ coincide, as expected. Moreover, the Dirac cone is observed around these energies. In case of $\mathrm{BL}$ graphene in ABAB.. stacking, a slight band gap opening is observed, Fig. 7(b).
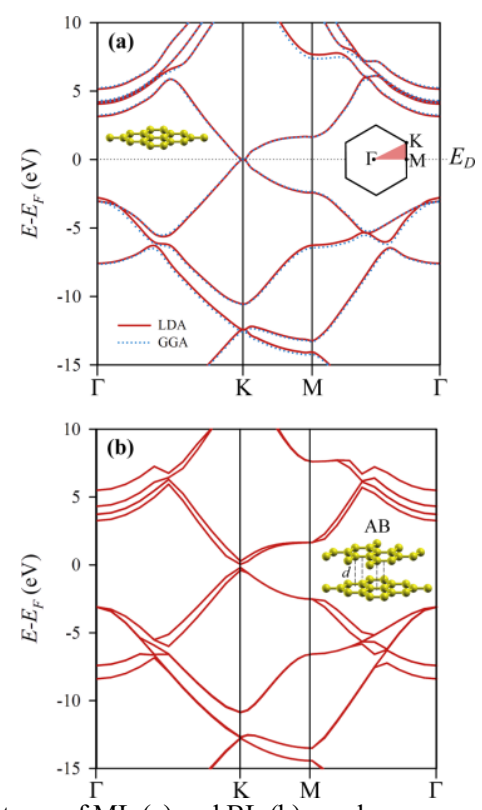

Fig.7. Band structures of ML (a) and BL (b) graphene.

The supercell employed in graphene-substrate calculations involves $\sqrt{3} \times \sqrt{3} R 30^{\circ}$ surface reconstruction in Fig. 2(a). It contains $18 \mathrm{Si}$ and $18 \mathrm{C}$ atoms belonging to the substrate and 8 $\mathrm{C}$ atoms belonging to the graphene superstrate. Fig. 8 shows that the first $C$ layer on the (0001) surface does not exhibit free-standing graphene behavior, where it forms a buffer layer. When the first $\mathrm{C}$ layer initial distance to the (0001) surface is $2.6 \AA$ or $3.0 \AA$, it approaches the surface upon relaxation, where the $\mathrm{C}_{\mathrm{G} 12}$ and $\mathrm{C}_{\mathrm{G} 17}$ atoms form mostly covalent bonds with the $\mathrm{Si}_{\mathrm{A} 12}$ and $\mathrm{Si}_{\mathrm{A} 13}$ atoms, Fig. 8(a). Besides, the $\mathrm{Si}_{\mathrm{A} 11}$ atom, whose dangling bond is not saturated, experiences less upwards displacement thant the $\mathrm{Si}_{\mathrm{A} 12}$ and $\mathrm{Si}_{\mathrm{A} 13}$ atoms. In case of the first $\mathrm{C}$ (buffer) layer, the value of the London $s_{6}$ parameter ( 0 or 1$)$ does not have significant effect on final relaxations on the (0001) surface.

The buffer layer in Fig. 8 is, on the average, separated from the top Si layer of the (0001) surface by $2.23 \AA$ and exhibits a buckling of $0.27 \AA$ ( $11 \%$ of graphene lattice constant). These results are close to the value $(2.58 \AA)$ reported by Mattausch and Pankratov [8]. Thus, the first $\mathrm{C}$ layer on the $6 \mathrm{H} \mathrm{SiC}$ (0001) surface is a buffer layer.

The second $C$ layer on the (0001) surface behaves like freestanding graphene, Fig. 8(b). The distance of this layer to the buffer layer is calculated as $3.39 \AA$ and $3.02 \AA$, respectively, for $s_{6}=0$ and 1 . Furthermore, the buckling in the second $\mathrm{C}$ layer is much less significant (around $2 \mathrm{pm}$ ). 


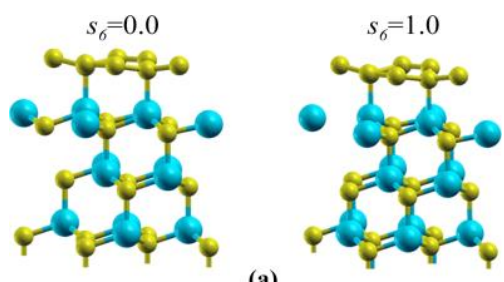

(a)

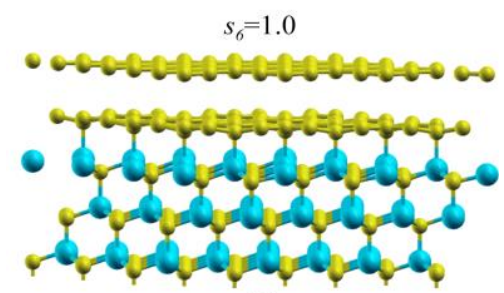

(b)

Fig.8. Final atom positions on the relaxed (0001) surface of $6 \mathrm{H} \mathrm{SiC} \mathrm{in} \mathrm{case} \mathrm{of}$ one (a) and two (b) C overlayers.

Band structures for one and two $\mathrm{C}$ overlayers on $6 \mathrm{H} \mathrm{SiC}$ (0001) surface are given in Fig. 9. Inclusion of dispersion forces by varying the London $s_{6}$ parameter does not have a significant effect on the obtained bands. The flat bands around $0.5 \mathrm{eV}$ in each case are related to the unsaturated dangling states of the substrate. Fig. 9(a) reveals that the graphene Dirac cone does not appear for the buffer layer. On the other hand, although overlapping with the substrate bands, a Diraccone like behavior is observed in Fig. 9(b) for two C overlayers.

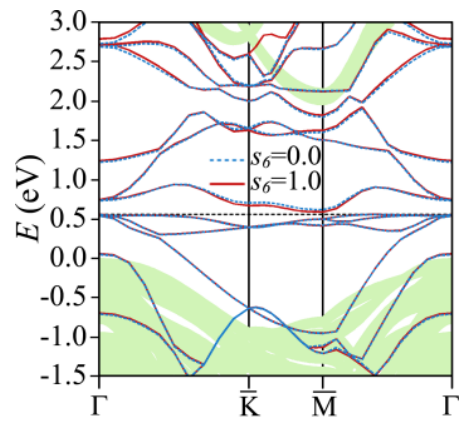

(a)

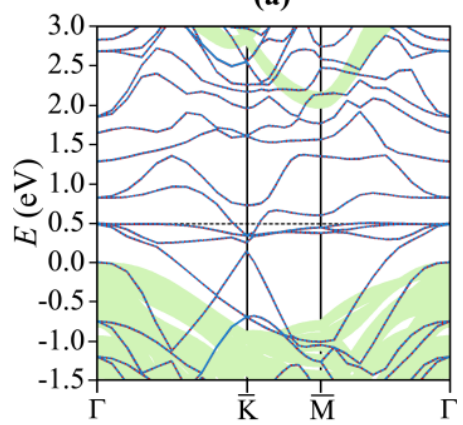

(b)

Fig.9. Band structure of one (a) and two (b) $\mathrm{C}$ overlayers on the $6 \mathrm{H} \mathrm{SiC}$ (0001) surface.

The final atomic positions for the geometrically optimized system of one $\mathrm{C}$ overlayer on the $(000 \overline{1})$ surface of $6 \mathrm{H} \mathrm{SiC}$ are given in Fig. 10(a). In contrast to Fig. 8(a), the first $\mathrm{C}$ layer is not bonded to the surface when London $s_{6}=0$. Thus, inclusion of the dispersion forces has a significant effect on the resulting

interactions. When $s_{6}=1$, the first $\mathrm{C}$ overlayer is covalentlybonded to the $(000 \overline{1})$ surface. The second case is similar to the results of Mattausch and Pankratov where local spin density approximation (LSDA) without explicitly considering dispersion forces $[8,23]$. In contrast, calculations by Magaud et al [25] on C-rich $(2 \times 2)_{\mathrm{C}}$ surface reconstruction of $4 \mathrm{H} \mathrm{SiC}$ suggest that the first $\mathrm{C}$ overlayer behaves like free-standing graphene. The latter is in agreement with experimental results and the DFT results for the $\sqrt{13} \times \sqrt{13} R 46.1^{\circ}$ surface reconstruction of $6 \mathrm{H} \mathrm{SiC} \mathrm{[46].}$

When $s_{6}=1$, The relaxations for the $(000 \overline{1})$ surface are significantly larger than those on the (0001) surface for both one $\mathrm{C}$ overlayer and two overlayers. The vertical displacement in the buffer layer is as high as $0.37 \AA$. Due to buckling in the buffer layer, the minimum bond angle is found to be $115.03^{\circ}$. The average distance between the buffer layer and the $(000 \overline{1})$ decreases to $2.02 \AA$. In contrast, the second $C$ layer behaves like free-standing graphene, Fig. 10(b), as in the case of (0001) surface in Fig. 8(b).

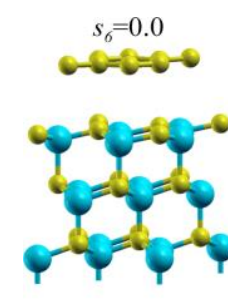

(a)
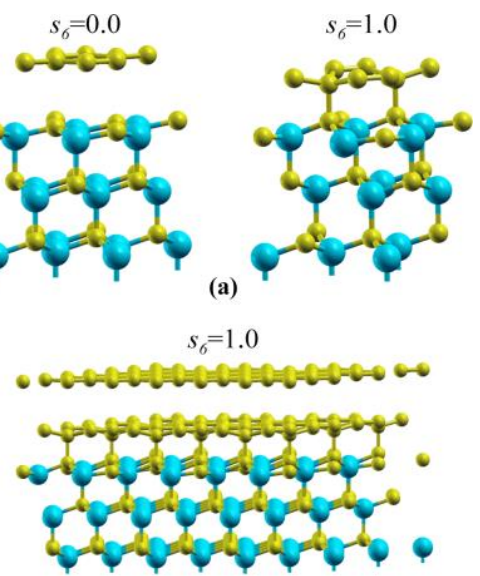

(b)

Fig.10. Final atom positions on the relaxed $(000 \overline{1})$ surface of $6 \mathrm{H} \mathrm{SiC}$ in case of one (a) and two (b) C overlayers.

The fluctuations in the first $\mathrm{C}$ overlayer when $\mathrm{s} 6=0$ are as low as $2.0 \mathrm{pm}$, where the $\mathrm{C}$ layers behave like free-standing graphene. The bond length in this layer does not significantly change from the initial value of $1.52 \AA$. Moreover, the graphene-substrate is measured as $2.94 \AA$.

The covalent bonding to surface on the right of Fig. 10(a), where the van der Waals forces are included in LDA calculations, stems from the nature of the numerical method and the van der Waals radii $\left(r_{0}=1.45 \AA\right)$ of the $\mathrm{C}$ atoms. While, the LDA calculations usually tend to over-bonding, the initial distance between the released $\mathrm{C}$ atoms is close to $r_{0}$. Thus, a tightly-bound atoms are obtained in these calculations.

The sharp contrast in Fig. 10(a) is also clearly visible in Fig. 11(a), where the band structure of one $\mathrm{C}$ overlayer on the $(000 \overline{1})$ surface is presented. While, a Dirac-cone like behavior, hallmark of graphene band structure, is observed for $s_{6}=0$, only four flat bands due the the buffer layer $\mathrm{C}$ atoms and unsaturated substrate $\mathrm{C}$ atoms appear for $s_{6}=1$. The discrepancy is also visible for two C overlayers, Fig. 11(b). 


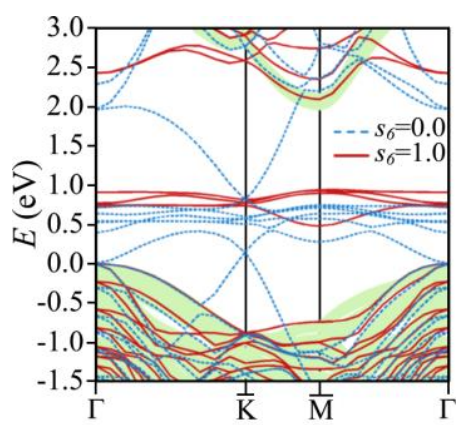

(a)

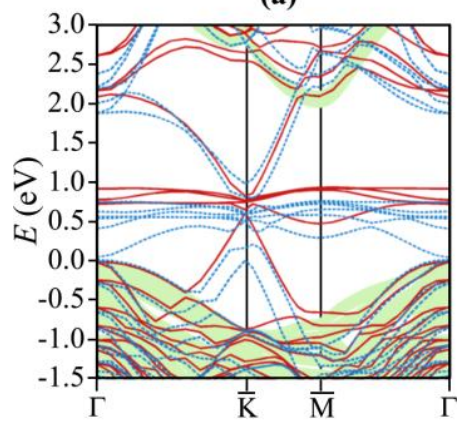

(b)

Fig.11. Band structure of one (a) and two (b) $\mathrm{C}$ overlayers on the $6 \mathrm{H}$ $\mathrm{SiC}(000 \overline{1})$ surface.

\section{CONCLUSION}

In conclusion, density-functional theory calculations of graphene on $6 \mathrm{H}$ silicon carbide $\{0001\}$ surfaces reveal that the free-standing graphene behavior is observed starting from the second carbon overlayer on either surface. In case of the Siterminated (0001) surface, the first $\mathrm{C}$ layer is a buffer layer, covalently bonded to the surface. This is the case irrespective of dispersion forces are accounted for. In contrast, the first $\mathrm{C}$ overlayer acts like a free-standing graphene layer on the $\mathrm{C}$ terminated $6 \mathrm{H} \mathrm{SiC}(000 \overline{1})$ surface when the van der Waals interactions between the overlayer and surface are neglected. However, intrinsic nature of LDA calculations and the van der Waals radii of $\mathrm{C}$ atoms lead to covalently-bonded first $\mathrm{C}$ overlayer (i.e. a buffer layer) when van der Waals interaction is taken into account. In all cases, both first $\mathrm{C}$ overlayers and the top substrate bilayer experience significant displacement from their equilibrium positions. However, the fluctuations on the second $\mathrm{C}$ overlayer is negligible. The results of this work can be used in estimating behavior of epitaxially-grown graphene few layers on $\mathrm{SiC}$ surfaces and developing their applications.

\section{ACKNOWLEDGMENT}

This work is supported by Akdeniz University Scientific Research Projects Coordination Unit under the grant number 2008.01.0105.010.

\section{REFERENCES}

[1] K. S. Novoselov, D. Jiang, F. Schedin, T. Booth, V. Khotkevich, S. Morozov, and A. K. Geim, "Two-dimensional atomic crystals," Proceedings of the National Academy of Sciences, vol. 102. 30, 2005, pp. 10451-10453.
[2] Z. Jiang, Y. Zhang, Y.-W. Tan, H. Stormer, and P. Kim, "Quantum Hall effect in graphene," Solid State Communications, vol. 143. 1-2, 2007, pp. 14-19.

[3] M. Katsnelson, K. Novoselov, and A. Geim, "Chiral tunnelling and the Klein paradox in graphene," Nature Physics, vol. 2. 9, 2006, pp. 620625.

[4] Y.-M. Lin, C. Dimitrakopoulos, K. A. Jenkins, D. B. Farmer, H.-Y. Chiu, A. Grill, and P. Avouris, "100-GHz transistors from wafer-scale epitaxial graphene," Science, vol. 327. 5966, 2010, pp. 662-662.

[5] P. Avouris, "Graphene: electronic and photonic properties and devices," Nano Letters, vol. 10. 11, 2010, pp. 4285-4294.

[6] K. Subrahmanyam, L. Panchakarla, A. Govindaraj, and C. Rao, "Simple method of preparing graphene flakes by an arc-discharge method," The Journal of Physical Chemistry C, vol. 113. 11, 2009, pp. 4257-4259.

[7] A. Kumar, A. L. M. Reddy, A. Mukherjee, M. Dubey, X. Zhan, N. Singh, L. Ci, W. E. Billups, J. Nagurny, and G. Mital, "Direct synthesis of lithium-intercalated graphene for electrochemical energy storage application," Acs Nano, vol. 5. 6, 2011, pp. 4345-4349.

[8] A. Mattausch and O. Pankratov, "Ab initio study of graphene on SiC," Physical Review Letters, vol. 99. 7, 2007, p. 076802.

[9] G. Giovannetti, P. A. Khomyakov, G. Brocks, P. J. Kelly, and J. Van Den Brink, "Substrate-induced band gap in graphene on hexagonal boron nitride: $\mathrm{Ab}$ initio density functional calculations," Physical Review B, vol. 76. 7, 2007, p. 073103.

[10] A. Reina, X. Jia, J. Ho, D. Nezich, H. Son, V. Bulovic, M. S. Dresselhaus, and J. Kong, "Large area, few-layer graphene films on arbitrary substrates by chemical vapor deposition," Nano Letters, vol. 9. 1, 2009, pp. 30-35.

[11] J. C. Swart, I. M. Ciob c , R. A. van Santen, and E. van Steen, "Intermediates in the formation of graphitic carbon on a flat FCC-Co (111) surface," The Journal of Physical Chemistry C, vol. 112. 33, 2008, pp. 12899-12904.

[12] L. Gao, J. R. Guest, and N. P. Guisinger, "Epitaxial graphene on $\mathrm{Cu}$ (111)," Nano Letters, vol. 10. 9, 2010, pp. 3512-3516.

[13] S. Marchini, S. Günther, and J. Wintterlin, "Scanning tunneling microscopy of graphene on Ru (0001)," Physical Review B, vol. 76. 7, 2007, p. 075429.

[14] P. Sutter, J. T. Sadowski, and E. Sutter, "Graphene on Pt (111): Growth and substrate interaction," Physical Review B, vol. 80. 24, 2009, p. 245411.

[15] D. Momeni Pakdehi, J. Aprojanz, A. Sinterhauf, K. Pierz, M. Kruskopf, P. Willke, J. Baringhaus, J. St ckmann, G. Traeger, and F. Hohls, "Minimum resistance anisotropy of epitaxial graphene on SiC," ACS Applied Materials \& Interfaces, vol. 10. 6, 2018, pp. 6039-6045.

[16] Z. Liu, Q. Xu, C. Zhang, Q. Sun, C. Wang, M. Dong, Z. Wang, H. Ohmori, M. Kosinova, and T. Goto, "Laser-induced growth of largearea epitaxial graphene with low sheet resistance on 4H-SiC (0001)," Applied Surface Science, vol. 514. 2020, p. 145938.

[17] X. Zhang, C. Yan, C. Zeng, T. Sun, Z. Xing, W. Shi, Y. Wang, C. Pang, and B. Zhang, "Epitaxial synthesis of graphene on $4 \mathrm{H}-\mathrm{SiC}$ by microwave plasma chemical vapor deposition," Materials Research Express, vol. 7. 11, 2020, p. 116410.

[18] N. Briggs, Z. M. Gebeyehu, A. Vera, T. Zhao, K. Wang, A. D. L. F. Duran, B. Bersch, T. Bowen, K. L. Knappenberger, and J. A. Robinson, "Epitaxial graphene/silicon carbide intercalation: a minireview on graphene modulation and unique 2D materials," Nanoscale, vol. 11. 33, 2019, pp. 15440-15447.

[19] T. Hu, H. Bao, S. Liu, X. Liu, D. Ma, F. Ma, and K. Xu, "Near-freestanding epitaxial graphene on rough $\mathrm{SiC}$ substrate by flash annealing at high temperature," Carbon, vol. 120. 2017, pp. 219-225.

[20] P. N. First, W. A. de Heer, T. Seyller, C. Berger, J. A. Stroscio, and J.S. Moon, "Epitaxial graphenes on silicon carbide," MRS Bulletin, vol. 35. 4, 2010, pp. 296-305.

[21] T. Cavallucci and V. Tozzini, "Intrinsic structural and electronic properties of the Buffer Layer on Silicon Carbide unraveled by Density Functional Theory," Scientific Reports, vol. 8. 1, 2018, pp. 1-10.

[22] C. Berger, Z. Song, X. Li, X. Wu, N. Brown, C. Naud, D. Mayou, T. Li, J. Hass, and A. N. Marchenkov, "Electronic confinement and coherence in patterned epitaxial graphene," Science, vol. 312. 5777, 2006, pp. 1191-1196.

[23] A. Mattausch and O. Pankratov, "Density functional study of graphene overlayers on SiC," Physica Status Solidi (b), vol. 245. 7, 2008, pp. $1425-1435$. 
[24] F. Varchon, R. Feng, J. Hass, X. Li, B. N. Nguyen, C. Naud, P. Mallet, J.-Y. Veuillen, C. Berger, and E. H. Conrad, "Electronic structure of epitaxial graphene layers on SiC: effect of the substrate," Physical Review Letters, vol. 99. 12, 2007, p. 126805.

[25] L. Magaud, F. Hiebel, F. Varchon, P. Mallet, and J.-Y. Veuillen, "Graphene on the C-terminated $\mathrm{SiC}(000$ 1) surface: An ab initio study," Physical Review B, vol. 79. 16, 2009, p. 161405.

[26] C. P. Huelmo and P. A. Denis, "Unraveling the electromagnetic structure of the epitaxial graphene buffer layer," Journal of Physics: Condensed Matter, vol. 31. 43, 2019, p. 435001.

[27] K. Hayashi, K. Morita, S. Mizuno, H. Tochihara, and S. Tanaka, "Stable surface termination on vicinal $6 \mathrm{H}-\mathrm{SiC}$ (0001) surfaces," Surface Science, vol. 603. 3, 2009, pp. 566-570.

[28] C. Park, B.-H. Cheong, K.-H. Lee, and K.-J. Chang, "Structural and electronic properties of cubic, $2 \mathrm{H}, 4 \mathrm{H}$, and $6 \mathrm{H} \mathrm{SiC}$," Physical Review B, vol. 49. 7, 1994, p. 4485.

[29] T. Seyller, A. Bostwick, K. Emtsev, K. Horn, L. Ley, J. McChesney, T. Ohta, J. D. Riley, E. Rotenberg, and F. Speck, "Epitaxial graphene: a new material," Physica Status Solidi (b), vol. 245. 7, 2008, pp. 14361446.

[30] K. Emtsev, F. Speck, T. Seyller, L. Ley, and J. D. Riley, "Interaction, growth, and ordering of epitaxial graphene on $\mathrm{SiC}\{0001\}$ surfaces: A comparative photoelectron spectroscopy study," Physical Review B, vol. 77. 15, 2008, p. 155303.

[31] R. Helbig and F. Engelbrecht, "SiC: Polar properties and their influence on technology and devices," in Advances in Solid State Physics 38: Springer, 1999, pp. 75-86.

[32] P. Giannozzi, S. Baroni, N. Bonini, M. Calandra, R. Car, C. Cavazzoni, D. Ceresoli, G. L. Chiarotti, M. Cococcioni, and I. Dabo, "QUANTUM ESPRESSO: a modular and open-source software project for quantum simulations of materials," Journal of Physics: Condensed Matter, vol. 21. 39, 2009, p. 395502.

[33] J. P. Perdew and A. Zunger, "Self-interaction correction to densityfunctional approximations for many-electron systems," Physical Review B, vol. 23. 10, 1981, p. 5048.

[34] J. P. Perdew, J. A. Chevary, S. H. Vosko, K. A. Jackson, M. R. Pederson, D. J. Singh, and C. Fiolhais, "Atoms, molecules, solids, and surfaces: Applications of the generalized gradient approximation for exchange and correlation," Physical Review B, vol. 46. 11, 1992, p. 6671.

[35] H. J. Monkhorst and J. D. Pack, "Special points for Brillouin-zone integrations," Physical Review B, vol. 13. 12, 1976, p. 5188.

[36] F. Birch, "Finite elastic strain of cubic crystals," Physical Review, vol. 71. 11, 1947, p. 809.

[37] G. C. Capitani, S. Di Pierro, and G. Tempesta, "The $6 \mathrm{H}-\mathrm{SiC}$ structure model: Further refinement from SCXRD data from a terrestrial moissanite," American Mineralogist, vol. 92. 2-3, 2007, pp. 403-407.

[38] W. Bassett, M. Weathers, T. C. Wu, and T. Holmquist, "Compressibility of $\mathrm{SiC}$ up to $68.4 \mathrm{GPa}$," Journal of Applied Physics, vol. 74. 6, 1993, pp. 3824-3826.

[39] M. Yoshida, A. Onodera, M. Ueno, K. Takemura, and O. Shimomura, "Pressure-induced phase transition in SiC," Physical Review B, vol. 48. 14, 1993, p. 10587.

[40] P. Käckell, B. Wenzien, and F. Bechstedt, "Influence of atomic relaxations on the structural properties of $\mathrm{SiC}$ polytypes from ab initio calculations," Physical Review B, vol. 50. 23, 1994, p. 17037.

[41] J. Wang, L. Zhang, Q. Zeng, G. L. Vignoles, and L. Cheng, "Surface relaxation and oxygen adsorption behavior of different $\mathrm{SiC}$ polytypes: a first-principles study," Journal of Physics: Condensed Matter, vol. 22. 26, 2010, p. 265003.

[42] J. Sołtys, J. Piechota, M. Łopuszyński, and S. Krukowski, "A comparative DFT study of electronic properties of $2 \mathrm{H}-, 4 \mathrm{H}$-and $6 \mathrm{H}-\mathrm{SiC}$ (0001) and $\mathrm{SiC}()$ clean surfaces: significance of the surface Stark effect," New Journal of Physics, vol. 12. 4, 2010, p. 043024.

[43] T. Dalibor, G. Pensl, N. Nordell, and A. Sch ner, "Electrical properties of the titanium acceptor in silicon carbide," Physical Review B, vol. 55. 20, 1997, p. 13618.

[44] J. P. Perdew, "Density functional theory and the band gap problem," International Journal of Quantum Chemistry, vol. 28. S19, 1985, pp. 497-523.

[45] M. Sabisch, P. Krüger, and J. Pollmann, "Ab initio calculations of structural and electronic properties of $6 \mathrm{H}-\mathrm{SiC}$ (0001) surfaces," Physical Review B, vol. 55. 16, 1997, p. 10561.
[46] J. Hass, F. Varchon, J.-E. Millan-Otoya, M. Sprinkle, N. Sharma, W. A. de Heer, C. Berger, P. N. First, L. Magaud, and E. H. Conrad, "Why multilayer graphene on $4 \mathrm{H}^{-} \mathrm{SiC}(000-1)$ behaves like a single sheet of graphene," Physical Review Letters, vol. 100. 12, 2008, p. 125504.

\section{BIOGRAPHIES}

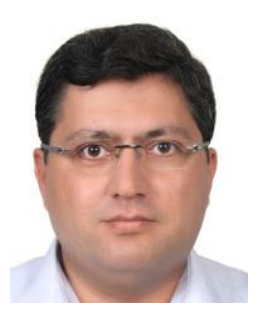

AHMET CICEK Finike, Antalya, in 1980. He received the B.S. degree in Physics from Bilkent University in 2003, M.S. and Ph.D. degrees in Physics from Akdeniz University in 2006 and 2012, respectively.

From 2005 to 2010, he was a Research Assistant with Physics Department of Akdeniz University. He was assigned as an Assistant Professor in the Physics Department, Burdur Mehmet Akif Ersoy University between 2014 and 2016. Later, he was assigned as an Associate Professor in the Department of Nanoscience and Nanotechnology, Burdur Mehmet Akif Ersoy University. He is currently working as a Professor at the same department since 2019. He is the author of three book chapters, 40 SCI articles, and 4 patent applications. His research interests include nanophotonics, plasmonic nanostructures, acoustic metamaterials, phononic crystals and numerical materials science.

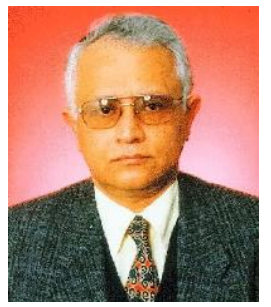

BULENT ULUG Yozgat, in 1953. He received the B.S. and M.S. degrees in Physics from Hacettepe University, in 1976 and the Ph.D. degree in Electrical Engineering from the University of Edinburgh, UK, in 1982.

He was Assistant Professor from 1983 to 1986 and Associate Professor from 1986 to 1996 in the Physics department, İn nü University. He has been a Professor with the Physics Department, Akdeniz University between 1996 and 2020. He is the author of more than 40 articles. His research interests include hightemperature superconductors, photonic and phononic crystals, quantum well structures and green synthesis of metallic nanoparticles. 